\title{
Physicians' Concept of Time Usage - A Key Concern in EPR Deployment
}

\author{
Rebecka Janols, Bengt Göransson, Erik Borälv, and Bengt Sandblad \\ Uppsala University, Department of Information Technology, Human-Computer Interaction, \\ Box 337, SE-751 05 Uppsala, Sweden
}

\begin{abstract}
This paper is based on an interview study with 19 resident, specialist and senior physicians. The study was initiated by a Swedish Hospital management to investigate physicians' attitude towards their EPR (Electronic Patient Records) and give recommendations for improvement in organization, development, deployment and training. The management had experienced that the physicians were unwilling to take part in the EPR deployment process and simultaneously complained about the low usability and potential safety risks of the systems. The study shows that the EPR must be considered a shared responsibility within the whole organization and not just a property of the IT department. The physicians must consider, and really experience, EPR as efficient support in their daily work rather than something they are forced to use. This includes considering work with the EPR as an important part of their work with patients.
\end{abstract}

Keywords: EPR, Physicians, Usability, Hospital Information Systems, Organizational Change, Deployment, Qualitative research, Management, Education.

\section{Introduction}

When implementing IT in health care it is important to have all potential users and management onboard. A large Swedish university hospital started to replace a paperbased record system with an EPR (Electronic Patient Record) system seven years ago. The system covers the whole care process, from primary care to specialized care at the hospital with totally 10000 employees. The EPR system makes it possible for all care professionals in the county, to track the patients through all health care activities, regardless of treatment unit. The system is modular and the deployment process has been step wise, with modules implemented successively.

In 2001, the county council started a process of investigating the needs of an EPR system for all their care units. One of the visions was to have one medical record per patient. This would enable shared care, meaning that all health care providers can access and register information related to a specific patient in the same record. In the process of choosing an EPR system, technical issues, safety and users' requirements were considered. Physicians, nurses, medical secretaries and other care professionals took part in the process as representatives from their professions. They had the opportunity to communicate their needs and requirements and evaluate the different 
systems. In 2003 the county council decided on a relatively new system from a Swedish company. They assumed that the system easily could be changed upon request and modified according to new needs and practices. Now, seven years after the first pilot deployment, the list of desired modifications has grown. Some of the users' requests have been implemented but there are still a lot to modify until the care professionals are satisfied with the system. Several other Swedish counties use the same EPR system and they all want their say in the development of the system. Therefore the process of modifying it is long and cumbersome. The systems customers are working together in a user group that negotiate with the company about future features and modifications to the system.

\section{Research Background}

Our research group conducts research in cooperation with several health care units of a Swedish University hospital since a couple of years. The overall method of the research project is action research, which means that we as researchers have dual aim with the research: "practical problem solving and generation of new knowledge" [1] . This paper describes a study where the purpose was to analyze the physician's attitudes toward EPR systems and the deployment process. It is the first interview study in a series with different care professions. The next study will be with nurses that work at the same units as the physicians. The result from these studies will later on be used as a basis for changes in the deployment activities.

\section{Purpose and Justification}

The hospital's EPR deployment group, the EPR support organization, the organizational system owner and other professions have expressed concerns with physicians not participating in the deployment process. Common remarks were that the physicians were not willing to participate in deployment activities, they were often complaining about the quality of the EPR systems and they seldom attended training sessions. The management described the physicians as a dominant profession and explained that the physicians' attitude was one of the reasons for the deployment process being cumbersome and the use of the EPR being inefficient. They tried to solve the problem by forcing them to take part in education sessions and other deployment activities. In order to investigate the reasons behind the experienced problems, we decided to make an interview study with physicians. The main purpose with the study was to clarify the physicians' attitude towards the EPR system, the deployment of the EPR and IT usage in general. Factors as the usability of the EPR were not in focus, although it is important that the EPR is intuitive, effective and supporting their needs.

\section{Related Research on Deployment of EPR and Physicians' Use of EPR}

A few studies have been made about physicians' use of and attitudes towards electronic medical records [2-7]. We have found some of them particularly relevant for our study. 
Marc Berg has done several studies on implementing information systems in health care organizations, e.g. [8].

Nancy Lorenzi et al. have studied organizational changes in health care organizations. Introducing systems in complex organizations requires more than technical skills. The challenges are often more behavioral than technical [9]

Robert H Miller and Ida Sims paper Physicians' use of electronic medical records: Barriers and solutions [4] is based on a qualitative study with physicians. They identify barriers such as high initial physician time costs, technology, difficult complementary changes, inadequate support, inadequate electronic data exchange and attitudes.

M.J. Van der Meijden et al. [7] have studied the users' role in design and implementation of an EPR system. Their methods were questionnaires and interviews. They came to the conclusion that both experienced and inexperienced users have little definite expectations regarding the effects of computers in health care. The only important aspects that the users mentioned were accessibility and reliability. Their conclusion is that "future users had no clear view of what could be expected after introducing computers into their daily work".

Ann-Britt Krog [10] has written a thesis on how care professionals negotiate with each other about how to use EPR systems. The thesis is based on qualitative studies at a Danish hospital. Krog mentions three common assumptions about EPR; better overview, less hazard and less time consumption. The studied users think that the EPR system gives them increased accessibility, increased communication and a better insight into each other's work between the professions. Her conclusion in the thesis is that the three mentioned visions have not been met in practice. As in the case above, we found these results interesting. The Danish and Swedish healthcare environment is comparable and the EPR used in Krog's study is actually the same that is used at the hospital we have studied.

\section{Method}

The main purpose of the study was to clarify the physicians' attitudes towards the EPR system, the deployment of the system and IT usage in general. To reach that purpose we decided to perform 19 semi-structured interviews with physicians with different level of expertise, age, and gender. The interviews were organized into the following six themes:

- Background information

- How EPR and other IT systems are being used

- Experience of the education and training process

- Participation in IT-development

- Experience of the IT-support in general

- The relationship between the physicians and the management

For each theme, the researchers started with a question and then continued with follow-up questions, more in a conversational style than as a formal interview.

The University hospital is divided into seven divisions with different specialties. The physicians were chosen from two of the divisions: Children and Women care and 
Emergency and Rehabilitation care. The selected physicians represented different levels of expertise, age and gender.

Two researchers performed the interviews during one month. The physicians were told that the purpose of the study was for the management to learn about the physicians' attitudes and experiences with regard to EPR and IT systems. The researchers explained that the interview was based on different themes and that the interview was going to last for 20-30 minutes. 12 of these were in this time slot, 3 become shorter and 4 became longer. The interviews were audio recorded and the researchers took notes in parallel.

\subsection{Analysis Method}

The interviews were planned and performed by the two researchers. All interviews were transcribed by one of the interviewing researchers. In the analysis phase a third researcher was introduced into the project. The researcher transcribing the interviews conducted the analysis together with this third researcher. The analysis method was affinity diagramming [11] with three iterations. The total amount of transcribed material was 160 pages. Each transcribed interview was marked with level of expertise, department, age and gender to enable deeper analysis. In the first iteration one third of the interviews were analyzed on paper. Interesting quotes were cut out of the paper, put on a wall and sorted into different categories without any predefined themes. (Figure 1)

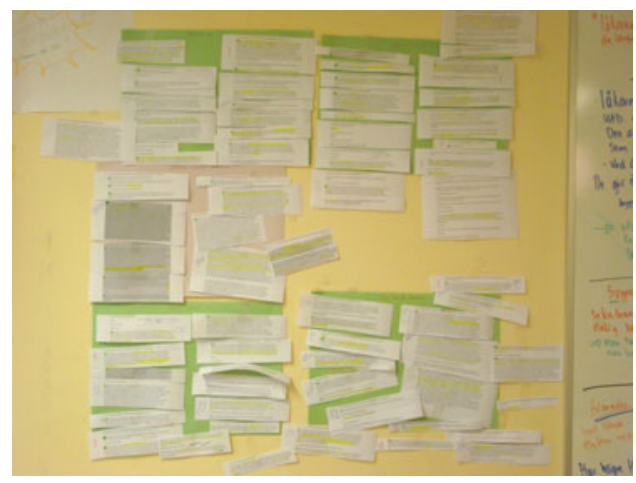

Fig. 1. The findings put on a wall in an affinity diagram

The quotes were then rearranged into named categories. In the second iteration the next third of the interviews were placed into the categories and then rearranged into new headlines. After that the last third of the interviews were analyzed and sorted into the named categories.

At this point we wanted to go deeper into the material. As the different groups of physicians have different responsibilities and work tasks, they also seemed to use the EPR system in slightly different ways and had mixed opinions about the usage of the system. The notes in each category were then rearranged according to three distinct groups, senior physicians, specialist physicians and resident physicians. We also 
gender marked physicians within the groups. But in our analysis, the expertise was far more significant than gender, so we decided not to go deeper into that analysis, although an interesting analysis might have been to look at all the material also with gender glasses.

\subsection{The Three Physician Groups}

The physician community is divided into different groups based on education level, research degree and expertise areas. There is a hierarchical difference between the groups that influence their status among physicians and other professions. (Table 1)

Table 1. The physicians divided into the groups with number of respondents, age and gender

\begin{tabular}{|l|l|l|l|}
\hline Group & Number of resp. & Age & Gender \\
\hline Resident & 5 & $27-34$ & 2 men 3 women \\
\hline Specialist & 8 & $34-55$ & 5 men 3 women \\
\hline Senior & 6 & $46-64$ & 5 men 1 woman \\
\hline
\end{tabular}

After basic education, the physicians do their residency at a hospital. They are called resident physicians and have been working at the hospital for 0-5 years. They work in one care unit for a longer time and they use the EPR for searching different kind of patient data that they use later on in the medical rounds. Their usage pattern is longer sessions with the EPR.

The second group is the specialists. They are specialists in different areas e.g. infection diseases, internal medicine or neonatology. They function as experts within their specialty and are doing a lot of different work tasks. They are moving between different care units within their medical specialty and are also working as consultant in different parts of the hospital. They are using the EPR for searching, writing, ordering tests and referrals and answering referrals. Their usage pattern is many short sessions with the EPR. Some of them are doing $\mathrm{PhD}$ research and others are doing their second specialty education.

The third group is senior physician. They all have a specialty and often a $\mathrm{PhD}$ degree. Most of the senior physicians in this group have management responsibilities, for a medical area or for other physicians. The seniors use the EPR system mainly for searching and not very frequently. They are concerned about that the EPR contains too much information, and consider it hard to find the important information.

\section{Findings and Analysis}

\subsection{Attitudes Toward IT and EPR}

Among the physicians there is a positive attitude towards IT and EPR systems in general. They are all quite used to computers and use them both at work and privately.

The physicians have huge expectations on how the EPR should help them and believe that the EPR systems give them benefits that the paper records could not give. 
For example, access to more patient information than before, quicker response on referral, possibility to have one complete record for a patient instead of several separate paper records. Despite the possibilities, the physicians are frustrated about the usability of the systems. The users feel that they are not in control because the system does not give a good overview, is not intuitive, requires a lot of "clicks" and involves many steps to perform the work tasks.

A senior physician expressed a view on the usability of the EPR that well shows the common attitude towards the EPR:

Interviewer:

"Would it be meaningful to modify the education sessions to be more tailored to your needs?"

Senior physician:

"No, the meaningful thing would be to make the EPR tailored to the users..."

Most of the physicians compare the electronic records with paper records but come to different conclusions. The senior physicians express that the EPR is much easier to use and talk about the differences between paper-based records and computer-based records. The paper records used to "disappear" and were not always readily available when needed. They believe that all systems have positive and negative aspects. They think that they are more positive towards EPR than other, younger physicians because they remember and compare it to the paper based systems.

A general attitude among the specialists is that the EPR system gives them some positive effects but it is not time efficient because of bad usability. They express their frustration, and some of them even claim that it is better to have some information on paper until they have an EPR that is fully supporting them in their work. The resident physicians come to the conclusion that computer records are safer than paper records. They too are frustrated with the system, but even if the systems are not fully usable they prefer the computer records over of a mix of paper and computer records, as it is today.

\subsection{Use of Time}

In general, the physicians seem to be continuously short of time. When they refer to time they use different conceptions of time e.g. patient time and administration time. The interviewed physicians argue that their time is more precious than other care professionals'. Medical knowledge, longer education and higher costs are arguments used. They argue that it is important that physicians not do tasks that other professions can do, otherwise it is a waste of time and money.

The physicians consider their time as split into two parts: patient time and administrative time. A general opinion is that their main work is done when they have direct patient contact. Other tasks, such as documenting, reading, ordering referrals and tests, are tasks that they consider to be done in administrative time. One specialist said:

"The drug module takes a lot of time from the patient time"

The main problem with this separation is that what they refer to as real "physician work" is only the tasks done during patient time. The tasks done in administrative time they refer to as tasks that they want to spend less time on. 


\section{"A lot of time [with the EPR] compared to the time I spend with the patients"}

The physicians on an average estimated their time in front of computers as $50 \%$ of their total time. According to their opinions they spend half of their time with tasks that they do not see as part of their "real work". If they would think of documenting, writing and reading referrals as a part of their "real physician work", it would probably affect their attitude towards EPR.

\subsection{Participation in Deployment Activities}

As mentioned above, the physicians refer to EPR and IT as something that is not a part of their "real work". Even though they are positive to IT in general, they are not motivated enough to participate in the deployment activities. A consequence of this is that other professions and the management experience that the physicians are unwilling to participate in optimizing EPR work. The senior physicians have all participated in different EPR development, requirement analyses, and taken part in formal groups or smaller informal groups, where they have discussed EPR and other IT questions. A common opinion is that they do not have the power to change anything in the systems. Some senior and specialists try to communicate their requests and thoughts but they express that the hospital management have created a structure that puts a distance between the care professionals and the IT organization. The specialists use lack of time/interests as well as lack of computer knowledge, as arguments for not participating. Some of them have taken part in EPR development activities, but they think that their participation was not valued highly enough. One specialist commented on her lack of participation:

"Yes, if you get time for it. I often think that they ask if you want to participate, but you need to participate instead of taking care of your patients or you have to postpone your consultations. And I don't think that is ok..."

On the other hand, some of the specialists say that when they express complaints about the systems, they are being listened to. But, changes to the systems take a long time to implement, and decreases their willingness to participate. When it comes to resident physicians they are not in such a position that they talk about participating in different EPR activities. They are often moving around between different departments and feel like they cannot educate older, more experienced colleagues. On the other hand, they think that they would have a lot to contribute because they are more used to computers.

To get improved participation, we think that the physicians need to find the EPR as "their" system, not as the IT organizations property.

Lorenzi [9] argue that it is a difference between implementing smaller systems and more complex systems into the organization. "It has become apparent in recent years that successfully introducing major information systems into complex health care organizations requires an effective blend of good technical and good organizational skills". Today the care staff and management in the health care organization consider the EPR as a technical system that is the IT organizations responsibility. We argue that the organization needs to change their attitude toward EPR, and look at EPR as the health care organization responsibility. This is of course not true for the technical 
infrastructure, but the implementation into the organization and how the system is used as a part of the care process. The IT organization shall be a support and IT expertise organization. If the EPR is the care organizations property, not the IT organizations, participation will be more appreciated and seen as more important.

\subsection{About the Education and Training Process}

When introducing new IT systems or EPR modules, the hospital uses an education model where representatives from each health care unit educate and teach their colleagues. A common opinion is that this education method is good because it gives an introduction to the systems.

"It is absolutely good that you have the possibility to go to education. But it is hard because the education-hour must be squeezed into the normal physician work. I know a lot of colleagues that missed the education because of lack of time, so they have had to learn the systems by themselves and in real-life situations."

The quote points out some main thoughts that are generally expressed by the physicians. The opinion is that the introduction is not supposed to be longer than 2 hours. Some even express that a 20 minutes briefing is enough. Longer education is considered a waste of time. The rationales for short intensive introduction differ between the groups. Here is a quote from a senior physician that is representative for their opinion:

"I always go to education sessions. But it cost too much to have one whole day for all physicians. I think that the system should be so good that you don't need any education at all, because everybody is using so many other IT systems. I had 20 minutes education at a physician meeting, you don't need any more. You need to know the system's possibilities and then learn to use it by yourself"

The quote indicates that the senior physicians prefer shorter education sessions because otherwise it would take up much too of a physician's time. It also illustrate that they think of education as an introduction to the system, not a session where they learn to use the system. The specialists think it will take too much of their own time. They will not be able to use that time for the usual patient and administrative work. Instead they have to deal with that later. The resident physicians' opinions can be summarized by this quote;

"Sometimes I think that the education sessions can be too basic and simple. They teach you things that you already know."

None of the physicians see any consequences from missing an education session. They all learn to use the system by using it in real-life situations, not at the education session.

Today the management handles the physicians' "negative attitude" by forcing them to go to education sessions and try to teach them how to use the EPR in the "right way". We think that today's point-and-click education session or viewing-the-system 
education is not the right way to achieve an attitude change toward the EPR. According to Berg, "In health care, however, the 'core business process' consists of highly knowledge-intensive, professional work, typified by a complexity that defies the predictability and standardization required for simple reengineering. Moreover, the professionals ultimately responsible for this process are powerful actors in the organization, and cannot be simply told to change their work patterns by senior management." [8] Therefore we considered the education ought to be role-based according to the different physicians work environment, needs and knowledge. The education should focus on how the EPR will support them in their work and not everything that is technically possible to do in the system. It is important that the education gives a critical perspective towards what the EPR can do for them [12], otherwise they get false expectations of what the EPR can support them in.

\section{Discussion}

The starting point for the study was to find out why physicians do not want to participate in the education sessions and other deployment activities. We conclude that this problem is more like the symptom than the diagnosis of the problem. The real problem is why the care professionals, physicians in this case, do not look at IT as a possibility to perform a better work. Why don't the physicians love their EPR system? The answer is complex. The separation between the EPR and the health care organization confirms the physicians' attitude that using the EPR is an administrative task, not something they want to spend time with. This attitude towards use of IT, which is one of their most important work tools, is not something we have seen in other professional areas. The EPR system is also something that the health care organization does not own and has the power and mandate to change to fit its needs.

Among the physicians, there is a mixed opinion about to which extent the EPR support their work, but the physicians agree on that accessibility is the best feature of an EPR. Beyond that the picture is unclear. We found some similarities with the Miller \& Sims study [4], for example difficulties with complementary changes, inadequate support and attitude. Differences between the studies are that Miller \& Sims focus on solo/small groups, while we focus on resident, specialists and senior physicians.

\subsection{The Differences between the Groups}

Residents have often worked with other EPR systems and are used to learn new IT systems. Their opinion is that the EPR has more advantages then disadvantages, but they are not expressing that the EPR is supporting them as much as it could. A general opinion among both specialists and senior physicians is that the system can be much more intuitive, usable and better in supporting them in their patient work. The difference between their opinions is that specialists are more disappointed and frustrated with the EPR, while the seniors understand that the EPR cannot be perfect in the beginning but have high hopes that the system will be better in the future. According to M.J. Van der Meijden et al. both experienced and inexperienced users have little defined expectations regarding the effects of computers in health care. The 
only important aspects that the users mention are accessibility and reliability. Meijdens' conclusion is that "future users had no clear view of what could be expected after introducing computers into their daily work" [7]. Our study indicates that the different physicians have different expectations concerning the benefits of an EPR. We believe that age, education, work tasks, work environment and responsibility are important aspects that explain the differences between the groups' attitudes. None of the physicians express that the EPR support them fully, but the specialists are most expressive and skeptical, because of their dependency of the EPR. The respondents in Meijdens' study expect that "an EPR should give them more overview than paper records and should release them from copying data from one sheet to the other" [7]. The respondents in our study do not think that the EPR gives better overview. In fact the specialists point out that using only paper records instead of a mix of papers and computers is better. Using mixed systems means lack of control, which makes them insecure about their ability to perform a great work, and that the EPR is too immature to be used without any complementary papers.

\subsection{Conception of Time Usage}

The groups have different work tasks and use their time differently. The residents are still learning to become physicians and are not conceptually separating EPR time and patient time as much as the specialists. The senior physicians have much more administrative tasks and are not using the EPR as much as the specialists. The conclusion is that the specialists are more protective about the patient time and hesitant to regard the EPR as a part of that time. We believe that the physicians, especially the specialists, ought to change their attitude toward EPR and should consider EPR usage as an integrated part of the total care work. Changing both the physicians and the health care organizations way of looking at EPR will support this.

\section{Summary}

The result of the analysis of the interviews, and our preliminary suggestions for improvements of organization and processes, can be summarized as follows.

The physicians have huge expectations on how the EPR should help them and believe that the EPR systems give them benefits that the paper records could not give. Despite the possibilities, the physicians are frustrated about the systems usability. The physicians consider their time as split into two parts: patient time and administrative time. A general opinion is that their main work is done when they have direct patient contact, they consider working in the EPR and other IT systems as administrative tasks. Their own estimation is that they spend $50 \%$ of their time in front of the computers.

The physician groups have different work tasks and different experiences with the system. They all have the possibility to participate in education sessions and find it useful as an introduction to the system. The residents think the systems are easy to use and think that the education sessions are too simple and basic. The specialists, on the other hand think that the systems have bad usability and find it better to keep some paper records until the EPR is safer and more intuitive. The seniors do not use the EPR as frequently as the other physicians. They are concerned that the EPR contains too much information, and consider it hard to find the important information. 
The seniors, and some of the specialists have participated in different development project but do not think that the hospital management listens to their opinions. We argue that it is important that the physicians participate in education sessions and deployment activities. If they do not participate in the education and training they will not learn to use their computer systems in an appropriate way and if they are not engaged in the development and deployment process they do not see the full potential of using EPR and other IT-systems to improve organization and work processes.

To solve this problematic situation, several changes must be made. Physicians' participation in development and deployment must be prioritized, given enough resources and be rewarded. They must see EPR and other IT-systems as the responsibility of the care organization and not of the IT department. Education and training procedures must be tailored to each target group and must be focused on learning the new way of working, not on how to handle the new technological artifact.

Acknowledgements. We would like to thank the county Council, hospital management and especially the physicians that managed to squeeze the interview into their tight schedule.

\section{References}

1. McKay, J., Marshall, P.: The dual imperatives of action research. Information Technology \& People 14, 46-59 (2001)

2. Likourezos, A., Chalfin, D.B., Murphy, D.G., Sommer, B., Darcy, K., Davidson, S.J.: Physician and nurse satisfaction with an electronic medical record system. The Journal of Emergency Medicine 27, 419-424 (2004)

3. Lærum, H., Ellingsen, G., Faxvaag, A.: Doctors' use of electronic medical records systems in hospitals: cross sectional survey. BMJ Journals 323 (2001)

4. Miller, H.R., Sim, I.: Physicians' use of electronic medical records: Barriers and solutions. Health Affairs 23(2), 116-126 (2004)

5. Varpio, L., Schryer, C.F., Lehoux, P., Lingard, L.: Working Off the Record: Physicians' and Nurses' Transformations of Electronic Patient Record-Based Patient Information. Academic Medicine 81 (2006)

6. Brown, S.H., Coney, R.D.: Changes in Physicians' Computer Anxiety and Attitudes Related to Clinical Information System Use. The Journal of the American Medical Informatics Association, 381-394 (1994)

7. Meijden, M.J.v.d., Tange, H., Troost, J., Hasman, A.: Development and implementation of an EPR: how to encurage the user. International Journal of Medical Informatics 64, 173-185 (2001)

8. Berg, M.: Implementing information systems in health care organizations: myths and challenges. International Journal of Medical Informatics 64, 143-156 (2001)

9. Lorenzi, N., Riley, R.T.: Managing Change: An Overview. Journal of the American Medical Informatics Association 7, 116-124 (2000)

10. Krog, A.-B.: Forhandlinger om patienten: den elektroniske patientjournal som kommunikationsmedie. Syddansk Universitet. Det Humanistiske Fakultet, Vol. PhD. Syddansk Universite, 204 (2009)

11. Beyer, H., Holtzblatt, K.: Contextual Design Defining Customer-Centered Systems (1998)

12. Ash, J.S., Berg, M., Coiera, E.: Some Unintended Consequences of Information Technology in Health Care: The Nature of Patient Care Information System-related Errors. Journal of the American Medical Informatics Association 11, 104-112 (2004) 